\title{
Odontogenic Infection Leading to Multiple Decompensations of Diabetic Ketoacidosis
}

\author{
Oumaima Elleuche ${ }^{1,2}$ Ines Kallel ${ }^{1,2}$ Eya Moussaoui ${ }^{1,2}$ Hana Bougatef ${ }^{1,2}$ Marouane Ouni ${ }^{1,2}$ Nabiha Douki ${ }^{1,2}$ \\ 'Department of Conservative Dentistry and Endodontics, Department \\ of Dental Medicine, EPS Sahloul, Sousse, Tunisia \\ ${ }^{2}$ Faculty of Dental Medicine, Oral Health and Oro-Facial Rehabilitation \\ Laboratory Research (LR12ES11), University of Monastir, 5019 Monastir, \\ Address for correspondence: Ines Kallel, Department of \\ Conservative Dentistry and Endodontics, Department of Dental \\ Medicine, EPS Sahloul, 4011 Sousse, Tunisia \\ E-mail: ineskallel@yahoo.fr
} Tunisia

\begin{abstract}
The aim of this case report was to highlight the association between Diabetic Ketoacidosis (DKA) and apical periodontitis through a case report involving a child suffering from DKA. The association between oral infections and diabetes has always been a debatable issue in the medical literature. Diabetic ketoacidosis (DKA) is a serious complication of uncontrolled diabetes. Oral infections, such as apical periodontitis is reported to be as a possible cause. Periapical lesions should initially be managed by a conservative non-surgical treatment. However, in cases of failure, endodontic surgery should be adopted. An 11-year- old child with type 1 diabetes under insulin therapy was hospitalized for DKA in 2019. A dentoalveolar abscess related to the upper right central incisor (11) was suspected as a possible cause of DKA. He had a history of trauma 3 years earlier followed by a coronal fracture of the 11. He also had an episode of DKA in 2018 and the same infection focus was identified as a possible factor but the patient did not finish his treatment. The radiograph showed a periapical lesion with radiolucency related to the open apex of the 11. Endodontic treatment was performed and the root canal system was obturated. The 3-month follow-up radiograph showed a favorable healing of the periapical lesion and the child had a stabilization of his diabetes.

Unfortunately, 2 months later, he was hospitalized for another episode of DKA. After exploration, the same dentoalveolar abscess related to the 11 was reported as a possible factor. The radiograph showed persistence of the periapical lesion. An endodontic surgery was therefore indicated. Twelve months later, complete healing of the periapical lesion was noted. The patient has not undergone an episode of DKA ever since.
\end{abstract}

Keywords: Diabetic ketoacidosis, endodontic surgery, endodontics, healing, periapical lesion, sinus tract

\section{Introduction}

Diabetic ketoacidosis (DKA) is the most serious complication of type 1 diabetes. It occurs in severe situations of insulin deficiency.[1] The major precipitating factors of DKA are infections and non-compliance with insulin therapy.[2] Oral infections, such as dentoalveolar abscess have previously been reported to be a possible cause of DKA.[2] They may occur preferentially in diabetic patients due to their immune system disorders. [3]

The association between diabetes mellitus and oral infections is very common. It is bidirectional and it is considered as a debatable issue in the medical literature. [3] It is therefore very important to establish awareness of the potential consequences of oral infections in dia-

How to cite this article: Elleuch O, Kallel I, Moussaoui E, Bougatef $\mathrm{H}$, Ouni M, Douki N. Odontogenic Infection Leading to Multiple Decompensations of Diabetic Ketoacidosis. J Pediatr Dent 2021;7(3):00-00 
betic patients to avoid diabetic ketoacidosis.[2] Oral infections are various; they could include apical periodontitis, periodontitis, and cysts[4] In fact, the development of apical periodontitis in endodontic practice results from root canal space infection. It is an inflammatory response of the host defense to the presence of microorganisms.[5]

All periapical inflammatory lesions should initially be treated with a conservative approach, even in cases of large periapical lesions. [6] Endodontic surgery is indicated as a final resort to save the endodontically treated tooth in cases of failure of the orthograde approach and persistence of the periapical pathology.[7]

We, herein, report a case of diabetic ketoacidosis secondary to a chronic apical periodontitis, which was treated by an endodontic surgery after the failure of the orthograde approach.

\section{Case Presentation}

An 11-year-old child, with a history of type 1 diabetes diagnosed in 2011 and receiving insulin treatment, was hospitalized in January 2019 in the pediatric department at Sahloul University hospital for decompensated diabetic acidosis. At presentation, he was febrile. He also presented with polyuria, polydipsia, blurred vision, vomiting, and severe dehydration. Treatment consisted of intravenous antibiotics (ampicillin and metronidazole), rehydrated with I.V normal saline together with insulin infusion. After full exploration, he was referred to our dental medicine department for management of a presumed odontogenic infection. A dentoalveolar abscess related to the right upper central incisor (11) was suspected as a possible cause of his decompensated diabetes.

External oral examination revealed no asymmetry or facial swelling. Interrogation of the patient revealed that he had a history of trauma 3 years earlier. He had a frontal impact on his incisors following a fall from his bicycle. According to his parents, he had an old episode of diabetic ketoacidosis in August 2018. He was then referred to the dental medicine department to manage a dental infection focus involving the same tooth, but he did not finish his treatment.

Intraoral examination revealed poor oral hygiene, and a productive mucosal sinus tract located $3 \mathrm{~mm}$ above the apex of the 11 (Fig. 1) presenting a coronal fracture with pulp exposure. It also revealed dental dyschromia, and negative pulp sensibility confirming the pulp necrosis. However, no painful apical percussion and no periodontal pockets were present.

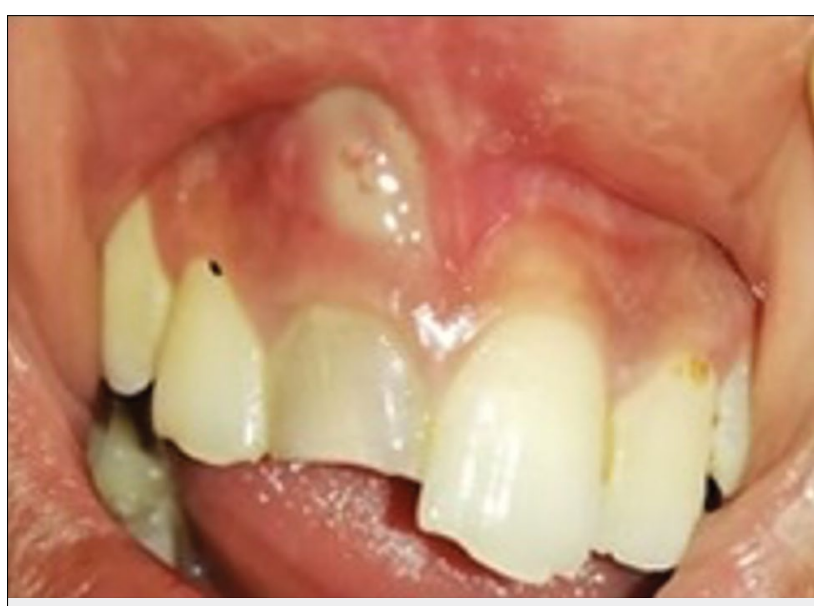

Figure 1. Mucosal sinus tract related to the tooth no 11

The sinus tract was traced with a gutta percha point, size 30. Periapical radiograph revealed an incomplete root development and a large periapical lesion, $5 \mathrm{~mm}$ in diameter, with uniform radiolucency and well-defined margins involving the wide-open apex of the 11(Fig. 2).

Based on the clinical and radiographic findings, diagnosis of chronic apical periodontitis complicated by a fistulized abscess was made. Conventional endodontic

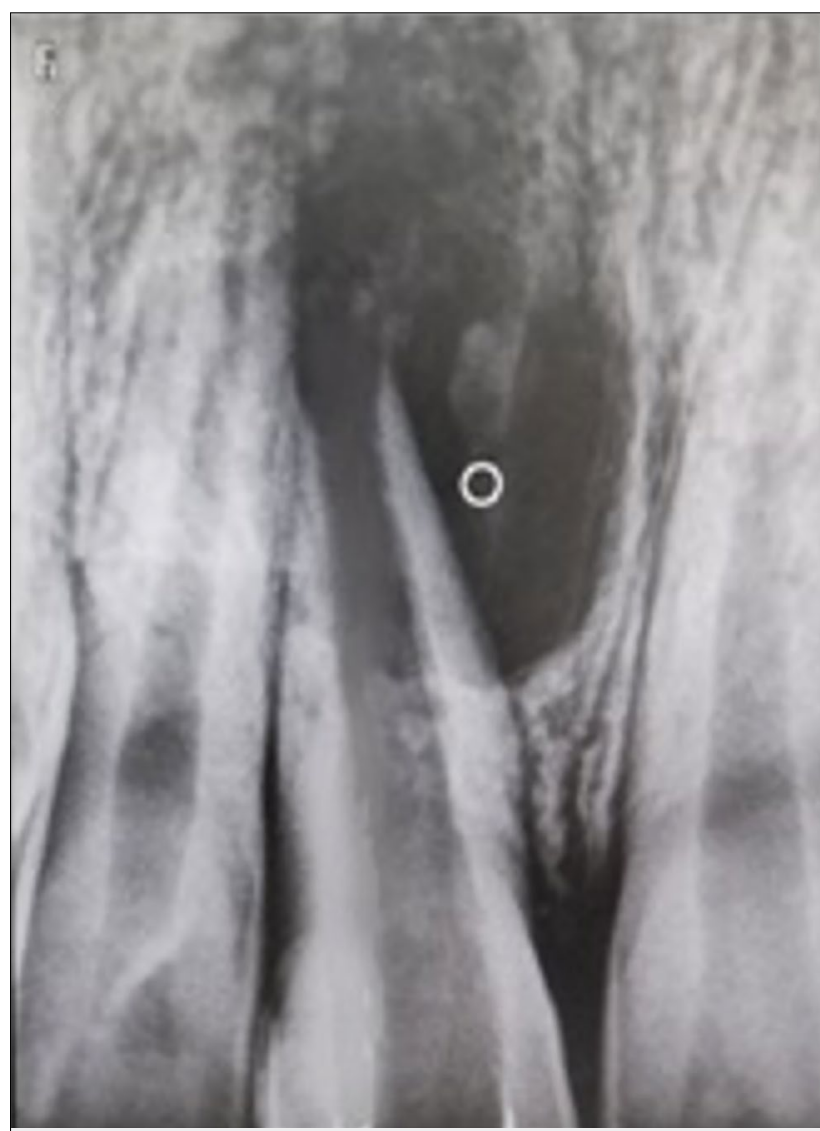

Figure 2. An immature tooth. Large periapical lesion $5 \mathrm{~mm}$ in diameter with uniform radiolucency 
treatment, being the first treatment option in cases of periapical lesions, was therefore planned. The conventional protocol of antibiotic prophylaxis for immunocompromised patients was respected ( $2 \mathrm{~g}$ of amoxicillin 1 hour before the act).

After setting the rubber dam and opening the access cavity (Fig. 3), the necrotic tissue was gently removed using K-Files 8, 10, 15, 20 at the working length $1 \mathrm{~mm}$ short of the radiographic apex (Fig. 4). The use of $\mathrm{K}$-files was limited to the purpose of debridement with no attempt to further enlarge the root canals. Slight drainage pus was observed during debridement. Copious irrigation with $2.5 \%$ sodium hypochlorite was performed. Dressing with Calcium hydroxide was used as an intra-canal medicament.

After 2 weeks, the root canal system was dried and obturated using a hydraulic condensation technique with a prefabricated cone and a bioceramic sealer (Bioroot ${ }^{\oplus}$, Septodont, Saint Maurdes-Fossess, France) to create an apical closure of the open apex (Fig. 5). Coronal filling was performed with composite resin.

Three days later, metabolic acidosis was resolved, and the child left the hospital. The patient was then scheduled for regular clinical and radiographic followup visits. The 3-month follow-up radiograph showed a favorable but not complete healing of the periapical radiolucency (Fig. 6).

Unfortunately, 2 months later, the child was hospitalized a second time for diabetic ketoacidosis, and he was referred to our dental medicine department to

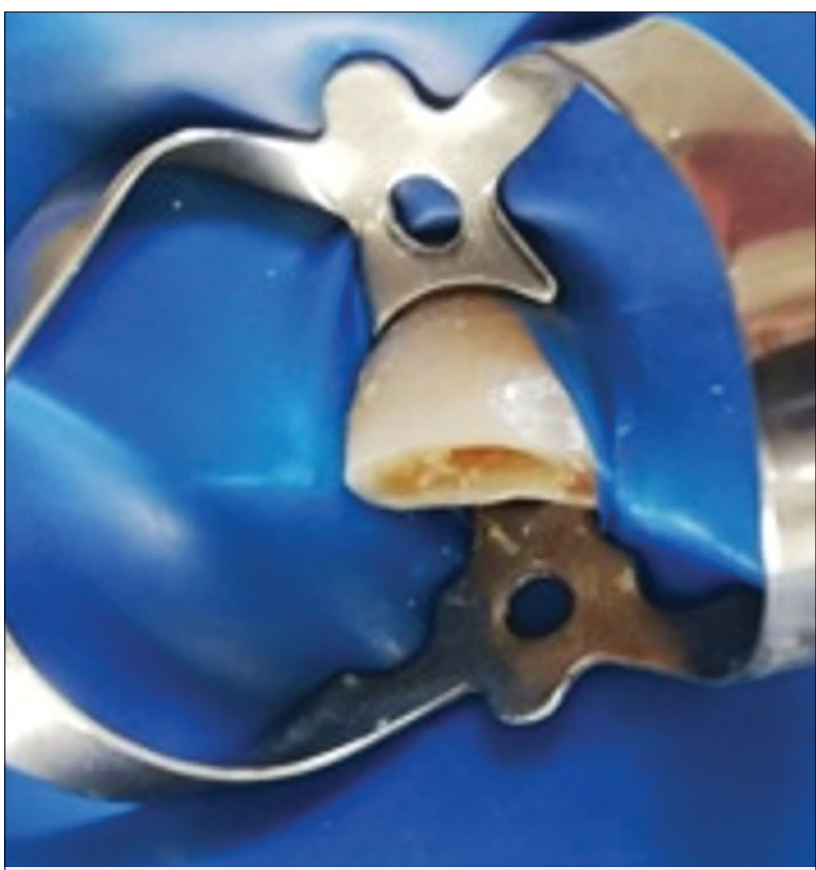

Figure 3. Rubber dam, access cavity

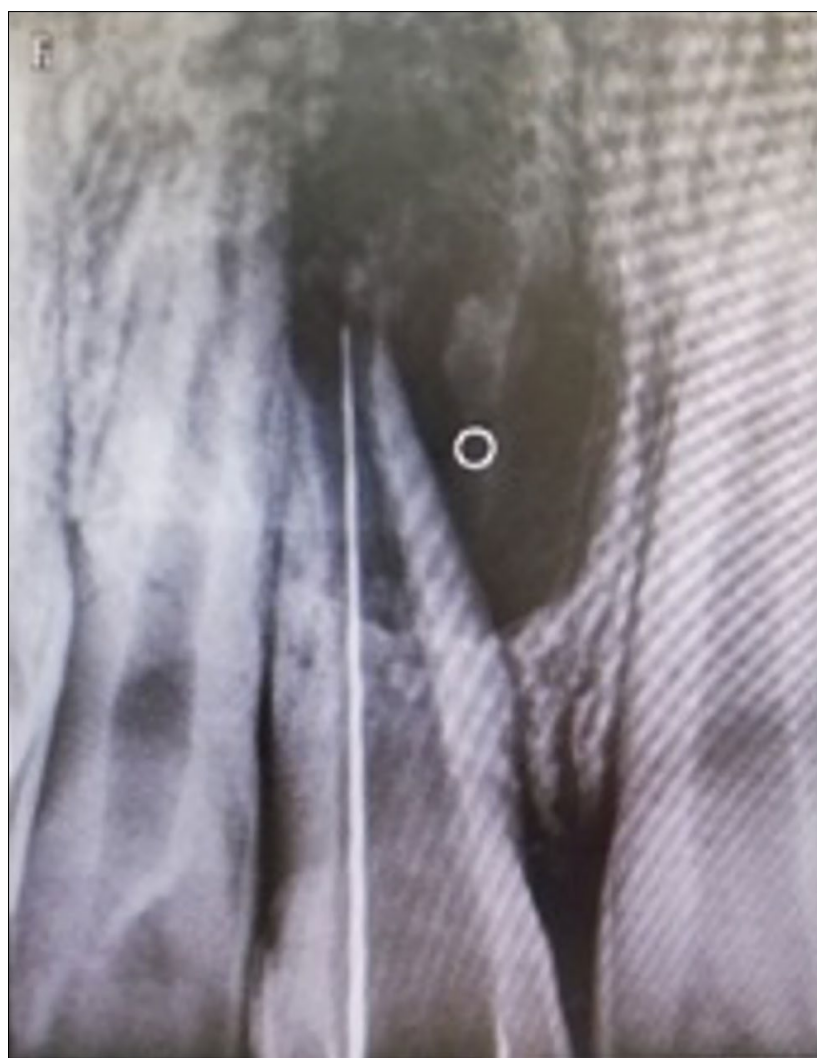

Figure 4. Radiograph showing the determination of the working length

search a dental origin. Intraoral examination revealed the reappearance of mucosal fistula related to the 11 (Fig. 7). Despite the successful endodontic treatment, retro-alveolar radiograph showed stabilization of the periapical lesion and a localized rounded opacity of 2 $\mathrm{mm}$ in diameter with a dental density into the lesion (Fig. 8). An endodontic surgery was therefore indicated as a final resort to explore and remove the periapical pathology. Following the administration of antibiotic prophylaxis ( $2 \mathrm{~g}$ amoxicillin 1 hour before), a local anesthesia was performed.

After elevation of the mucoperiosteal flap, fenestration of the vestibular bone table was detected (Fig. 9) and curettage of the granulation tissue was performed. The detected opacity into the lesion corresponded to a rounded mass without sharp edges, which was in favor of an odontoma or a fractured dental fragment (Fig. 10). After that, osteotomy was performed to enlarge the bony defect to a buccal window (Fig. 11).

Straight fissure carbide bur was used with full rotating power to ensure resection of the $3 \mathrm{~mm}$ of the apex. Saline irrigation was used to clean the cavity and to remove the debris of gutta-percha, dentin, and cementum. Then, sterile gauzes soaked in anesthetic with vasoconstrictor were gently placed inside the empty cavity to obtain 


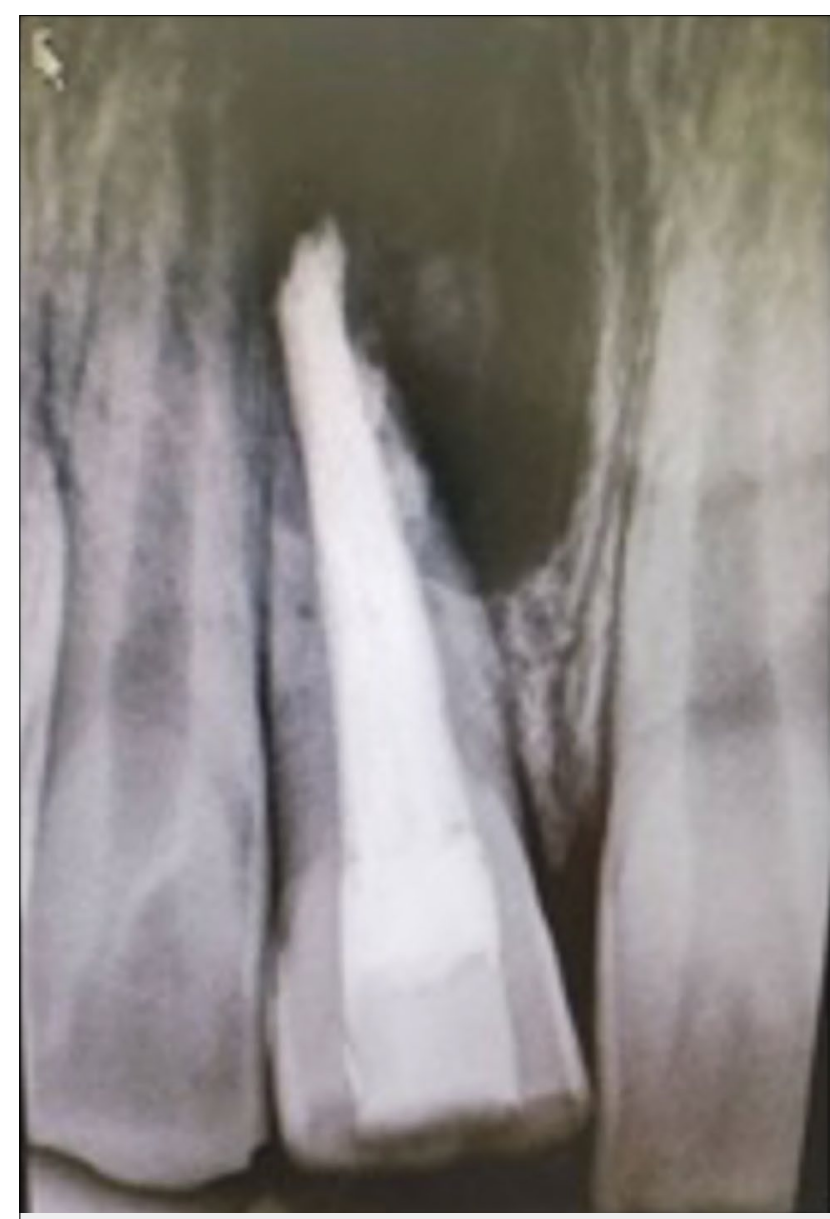

Figure 5. Post-operative radiograph

hemostasis. A retrograde preparation with ultrasonic tips was performed and root end filling was obtained with intermediate restorative material (IRM) (Fig. 12). Later, the mucoperiosteal flap was sutured (Fig. 13) and post operating radiograph was conducted (Fig. 14). Anatomopathological examination of the operating piece was carried out but the result was not conclusive.

1 week later, the child had a stabilization of his diabetes. He left the hospital and regular follow-up visits were scheduled. Three months later, the follow-up radiograph showed favorable healing of the periapical lesion (Fig. 15). The follow-up radiograph at 12 months revealed almost a total healing of the periapical lesion (Fig. 16). Ever since, the patient has not undergone a decompensation episode of his diabetes.

\section{Discussion}

Diabetic Ketoacidosis (DKA) is a serious complication of diabetes representing an extreme emergency in the spectrum of decompensated diabetes mellitus.[8] It has a mortality rate varying between 5 and $10 \%$ and it rep-

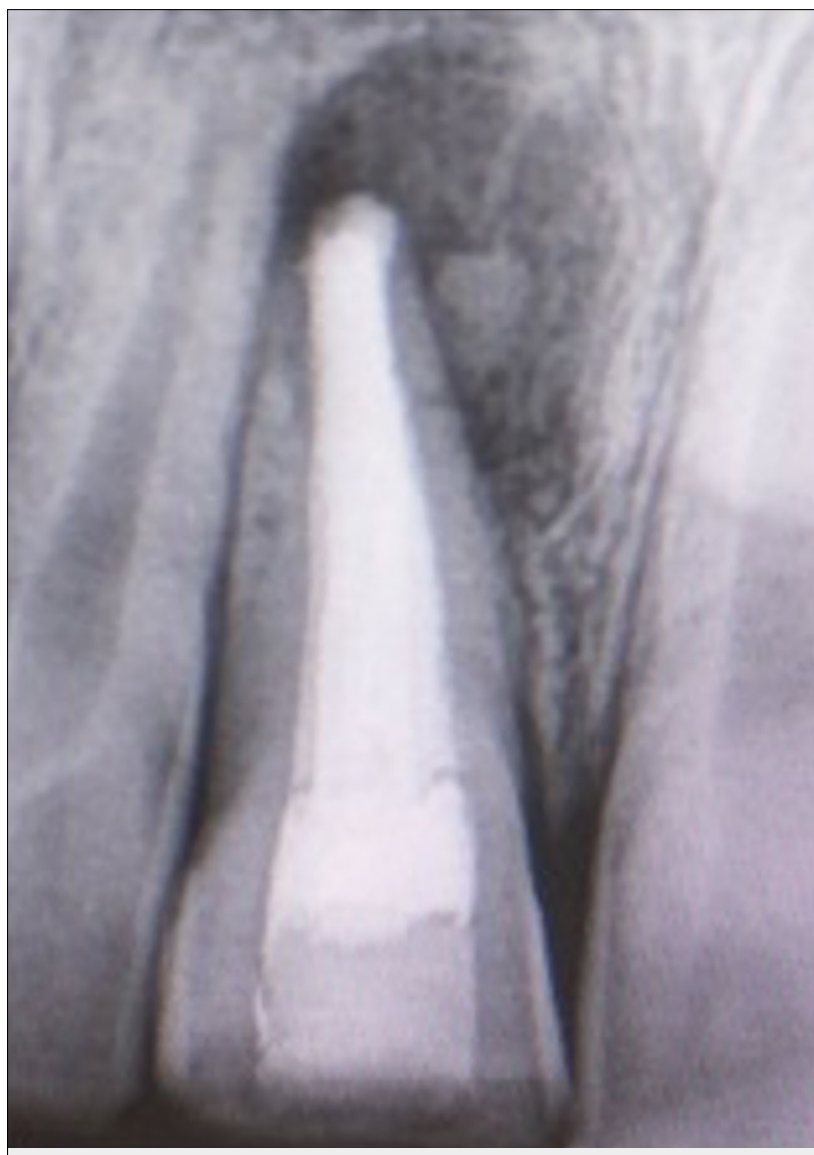

Figure 6. Follow-up radiograph 3 months later

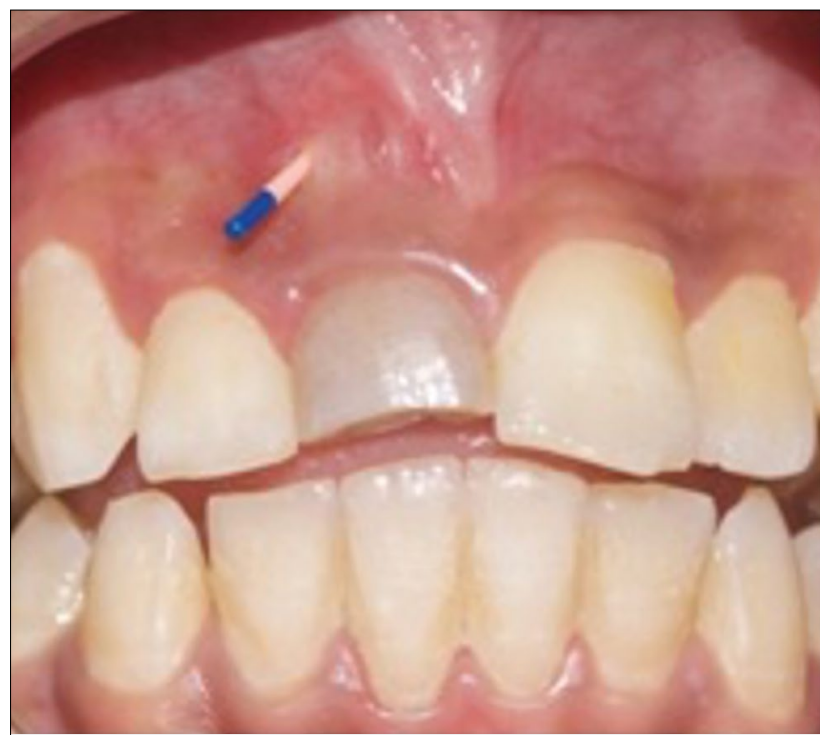

Figure 7. Reappearance of the mucosal sinus tract related to the tooth no 11

resents the major cause of death in children with diabetes mellitus (DM).[9] DKA is most common among patients with diabetes type 1 although it can be seen in patients with diabetes type 2.[8] 


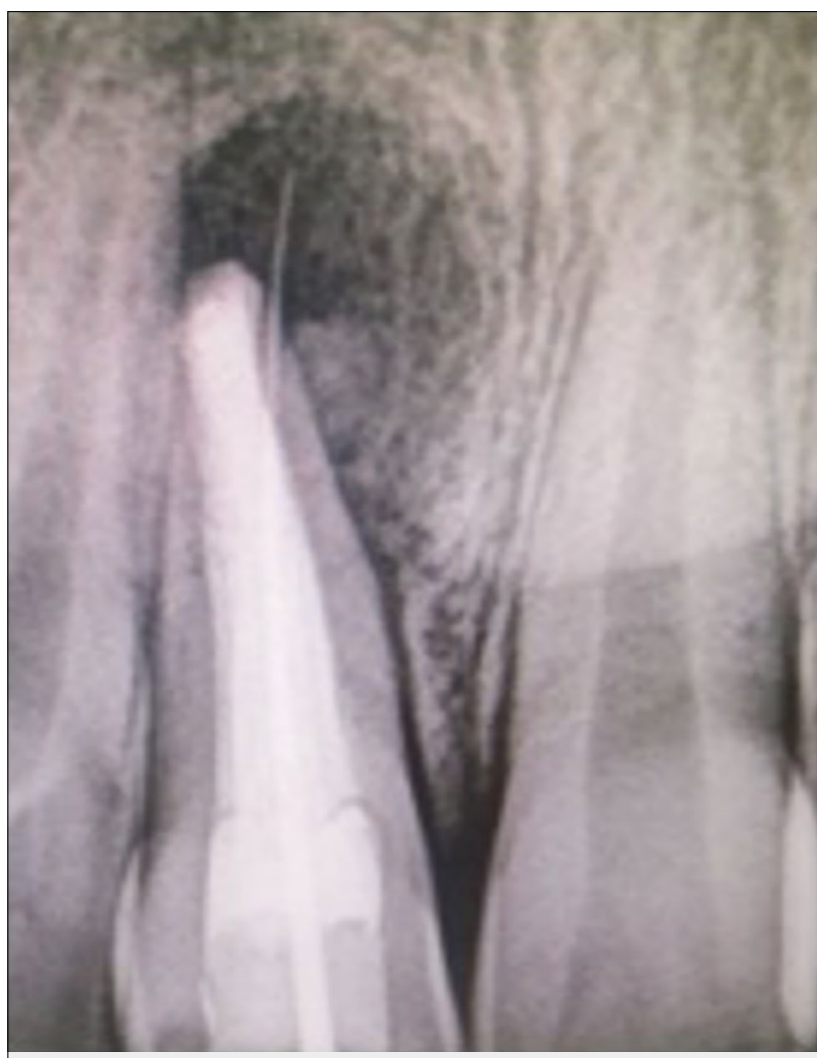

Figure 8. Radiograph showing persistence of the periapical lesion, a localized $2 \mathrm{~mm}$ rounded opacity with dental density into the lesion

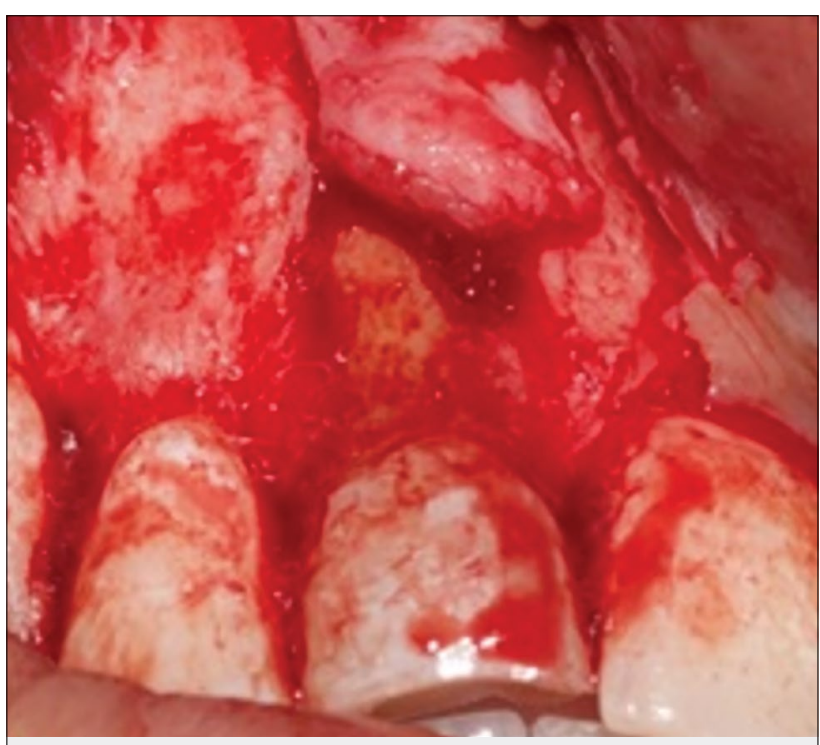

Figure 9. Flap elevation and fenestration of the vestibular bone

The major clinical symptoms are dehydration, polyuria, polydipsia, nausea, vomiting, abdominal pain, and an eventual confusion.[10] The classic triad of DKA are Hyperglycemia, excessive production of ketone bodies, and metabolic acidosis resulting from prolonged

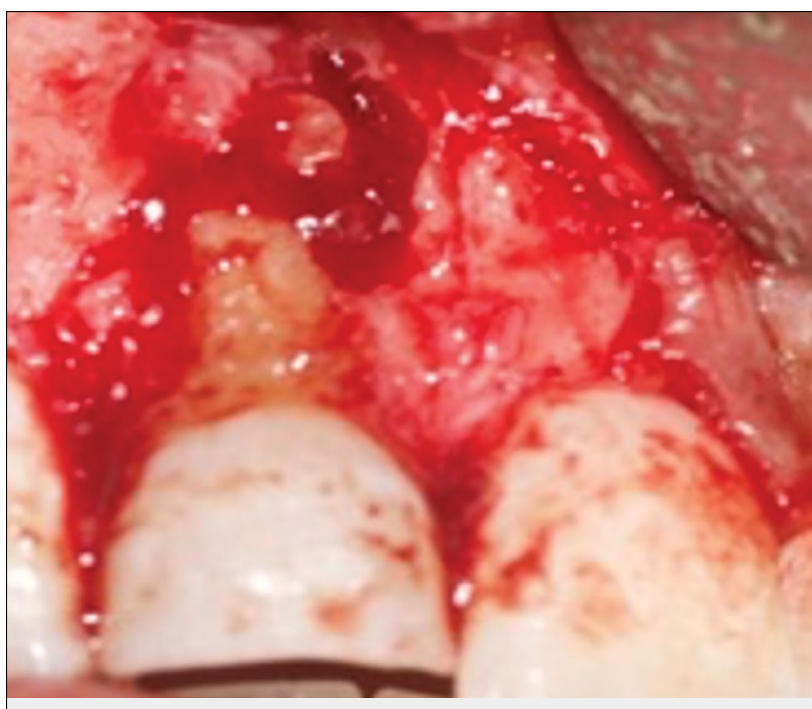

Figure 10. The rounded mass in favor of an odontoma

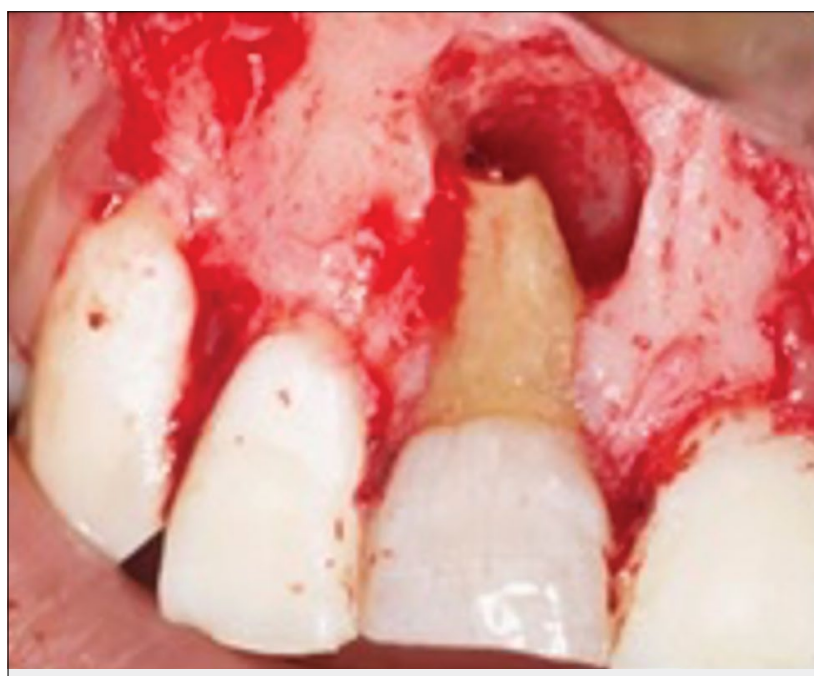

Figure 11. Osteotomy

absence of insulin representing the underlying pathophysiologic cause.[9]

Treatment of Ketoacidosis includes fluid replacement, insulin therapy, and correction of electrolyte disturbances to decrease serum glucose concentrations and to clear serum ketone.[8] Our patient was hospitalized for severe dehydration with vomiting, polyuria, and polydipsia. He was under insulin infusion, IV normal saline, and intravenous antibiotics.

The most frequently reported precipitating factors are infection and non-compliance with insulin therapy. [8] Pancreatitis, myocardial infarction, use of medication having a hyperglycemic effect such as steroids or diazoxide, and pregnancy are also described as possible causes.[8]

Oral infections are reported to be a cause of DKA. The link between periodontal disease and diabetes is 


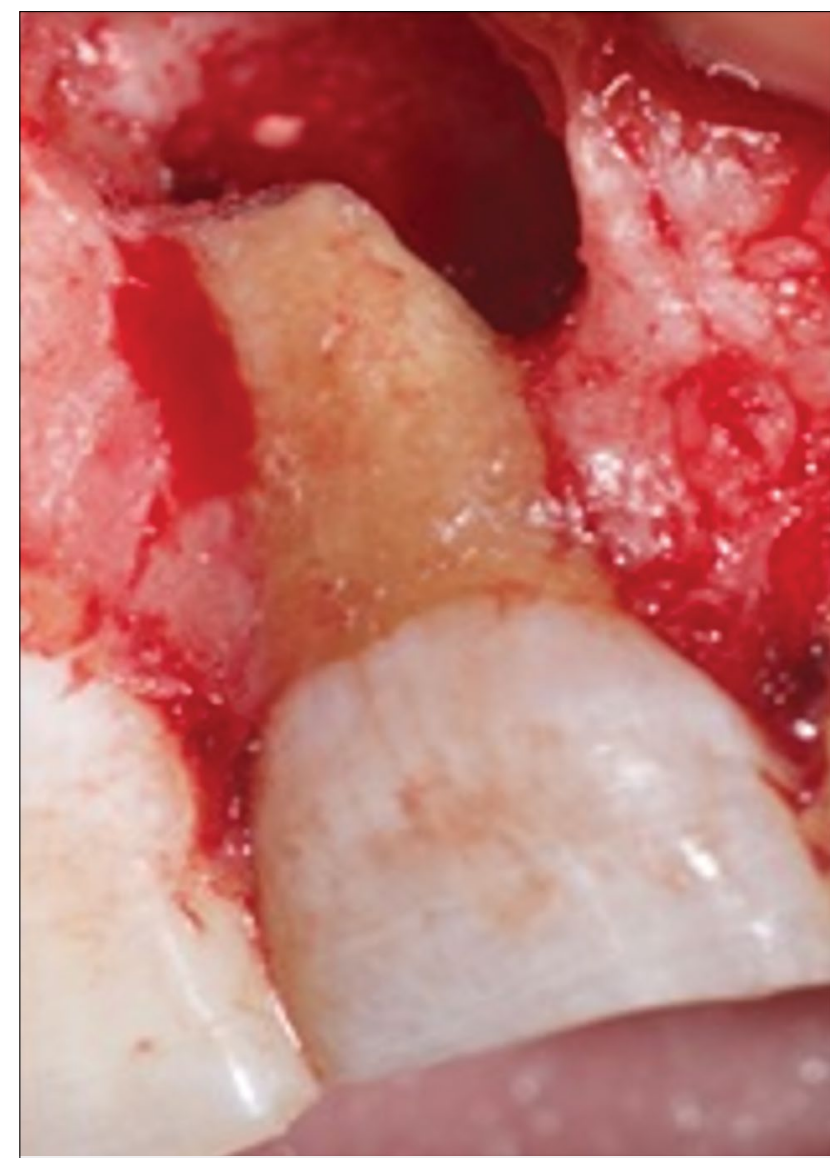

Figure 12. Root end obturation with IRM

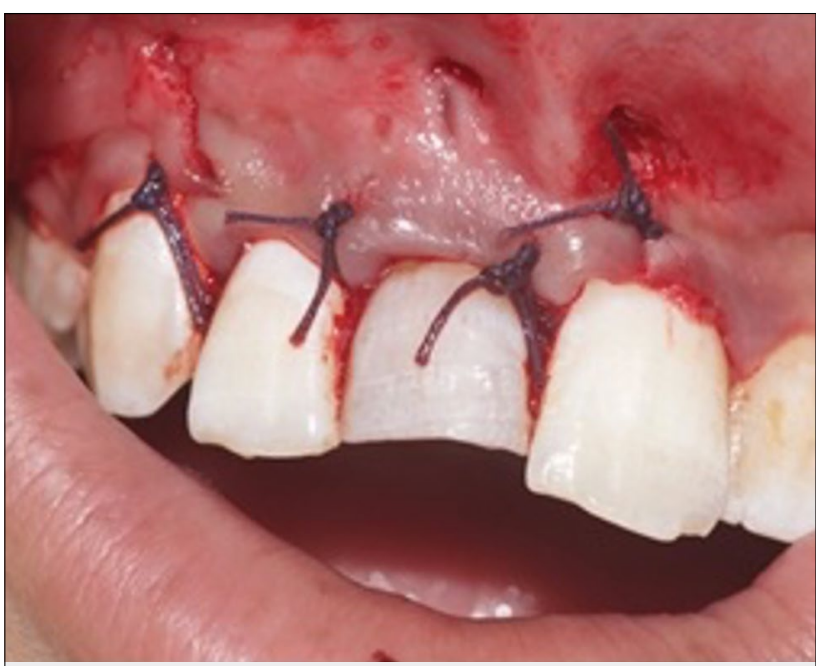

Figure 13. Sutures

well-documented. However, apical periodontitis is also considered a possible risk factor of DKA.[2] Dentoalveolar abscess has previously been reported to be a precipitating cause of DKA. Dentists should therefore be aware of the potential consequences of oral infection in a diabetic patient and should treat oral diseases as earlier as possible to avoid DKA. Studies have

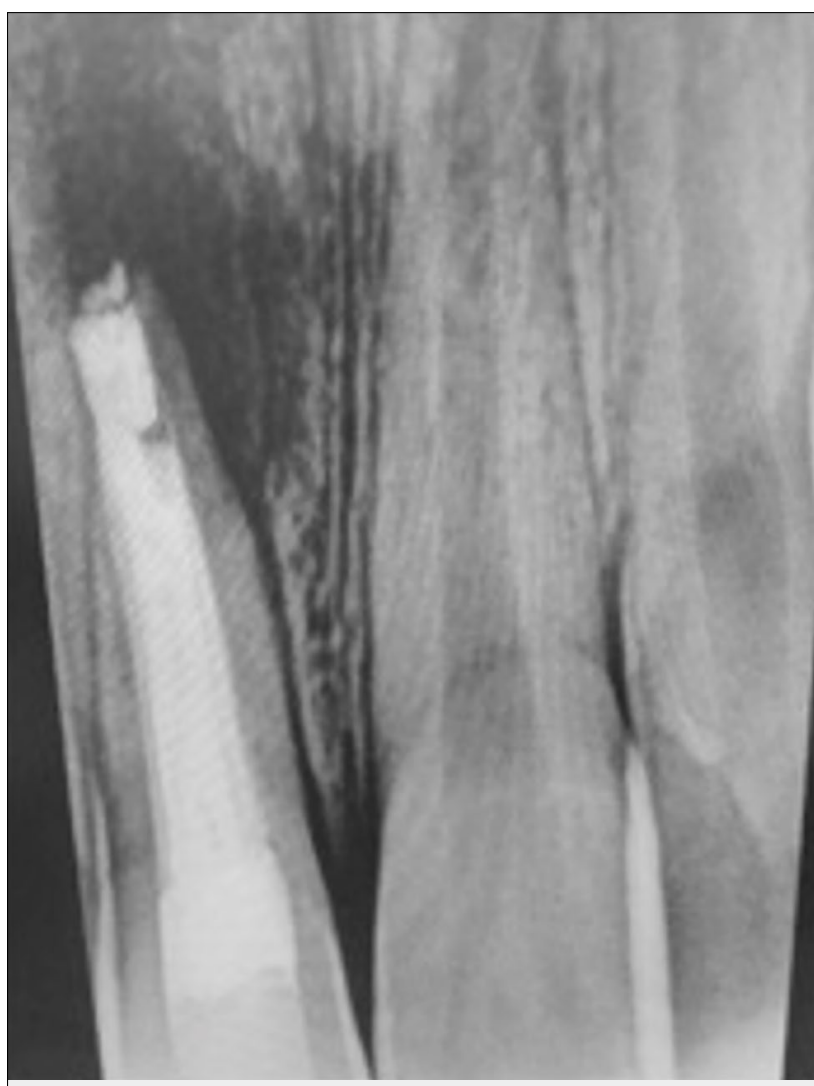

Figure 14. Post-operative radiograph

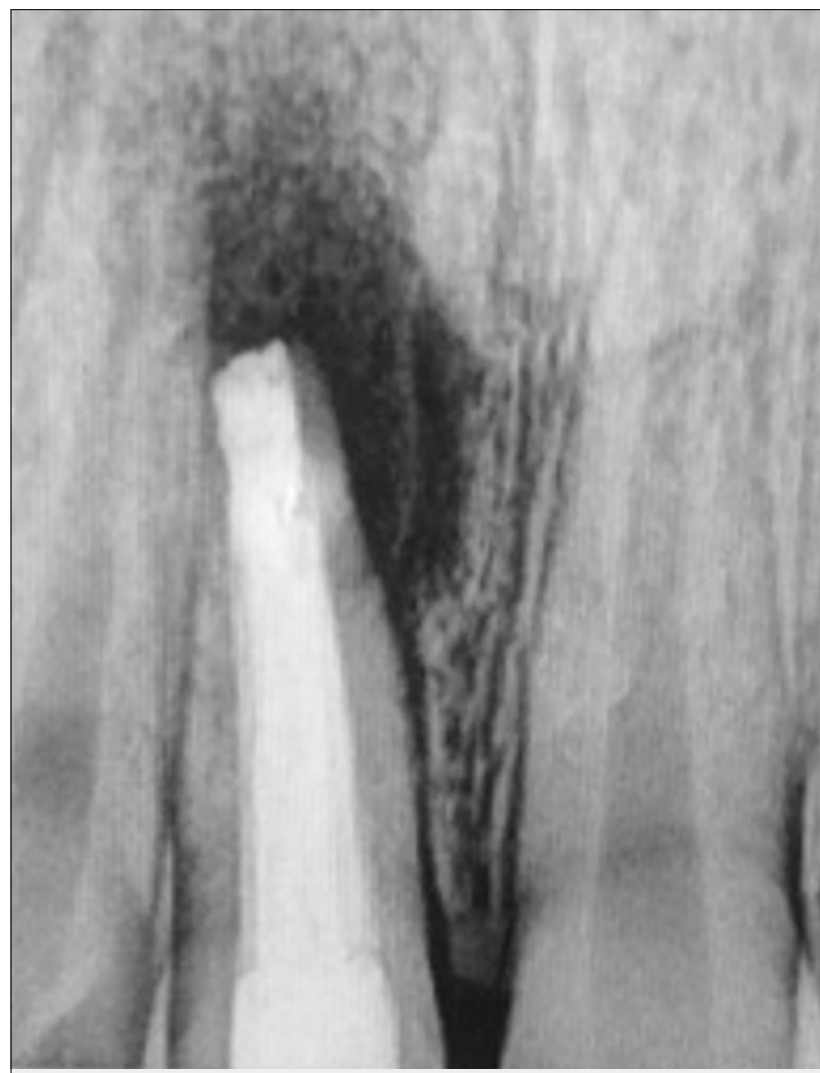

Figure 15. Follow-up radiograph 3 months later 


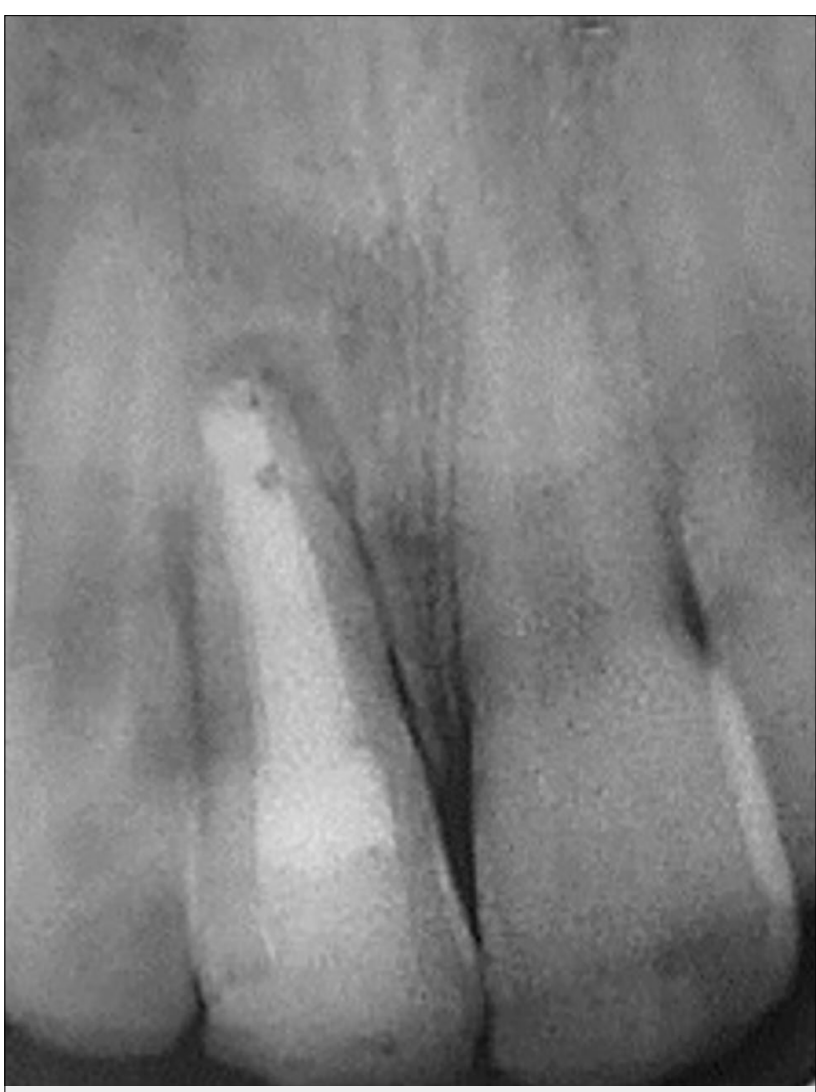

Figure 16. Follow-up radiograph 12 months later

revealed that undiagnosed oral infections in diabetic patients may lead to an extreme situation of emergency. [2] So, the connection between diabetic decompensation and dental infections is very common and it is bidirectional. It is currently considered the most interesting aspect faced by the medical and dental scientific community.[3]

To validate this association, biologically plausible mechanisms must be evident to explain the pathobiology of this interaction.[10] In fact, apical periodontitis is an inflammatory process around the apex of a tooth root. It is a sequel to microbial infection of the pulp space. It causes a number of local tissue responses to limit the spread of the infectious elements, which may not be a local phenomenon.[11] Several recent studies have discussed the relationship between endodontic disease and diabetes. They proved that focal oral infection may lead to a systemic health disease.[12] Although periapical periodontitis induces local phenomenon, they can cause the spread of the infectious elements to nearby tissues compartments which may lead to fatal inflammatory conditions. [5] So, an infected root canal may act as a focus of infection to distant body sites. A connection of lipopolysaccharide from anaerobic gram negative bacteria causing apical periodontitis with Toll- like receptor (TLR4) on macrophages and neutrophils activates the broad axis of the innate immunity (cytokines, prostaglandins..). These cytokines may be released into the systemic circulation, inducing a systemic inflammatory status which can promote an increase in overall insulin resistance and altering the metabolic control in diabetic patients leading to diabetic complications, such as the DKA.[11]

Studies by Bender et al also proposed that increased local inflammation and elevated cytokine levels of apical periodontitis cause a rise in blood glucose and an intensification of diabetes, placing patients in an uncontrolled diabetes and making them more susceptible to develop DKA.[12] The relationship between diabetes and oral infection is considered as a vicious circle because diabetes may also lead to dental infection. Innate immunity, hyperglycemia, and high serum levels of advanced glycation would predispose to chronic inflammation. Once oral infection is established, it may exacerbate systemic disease progression.[12] Patients with diabetes mellitus may have defects in adherence, chemotaxis, phagocytosis, and in the antioxidant, activity involved in bacterial function.[12]

In fact, diabetes may induce changes in immune cell functions. Up-regulation of inflammatory cytokines from monocytes and leucocytes, and down-regulation of growth factors from macrophages may facilitate the spread of local periapical infection in systemic circulation and may be the cause of DKA.[11] Eradication of odontogenic infection is necessary and should be performed as earlier as possible to establish diabetic stability.

All inflammatory periapical lesions of endodontic origin should always be initially treated with a conservative approach whatever the size of the lesion is. Indeed, if successfully performed, conventional endodontic treatment by orthograde route, which is less invasive, can initiate the healing process.[10] Studies have reported a success rate of up to $85 \%$ of complete and partial healing of periapical lesions after non-surgical endodontic treatment. [10]

In our case, the patient was immunocompromised. He had episodes of DKA, and his trauma dated back to 3 years. Thus, due to the immune system disorders, it could have been better if surgical management of the periapical lesion was indicated at the onset to ensure better healing and to avoid any risk of complications or a failure of the orthograde approach.

During endodontic treatment, appropriate irrigation should be established. The sodium hypochlorite 
$(\mathrm{NaOCl})$, ethylenediaminetetraacetic acid (EDTA), chlorhexidine (CHX), and other irrigation solutions help to reduce the microbial flora of the infected canals. Ultrasonic activation increases their efficiency. [10] In addition, the use of calcium hydroxide as an intracanal medication after chemo-mechanical preparation provides a better chance of periapical healing. In fact, if performed alone, mechanical preparation does not reach the lateral root canals and the dental tubules.[10]

Root canal treatment in a single visit is also possible and may have favorable outcome in case of a dry root canal.[5] Adequate three-dimensional obturation of the root canal is one of the keys to non-surgical treatment success.[13] In our case, root canal disinfection was ensured using $2.5 \% \mathrm{NaOCl}$ that was activated with a gutta percha master cone. Since the canal was not dry, calcium hydroxide was placed for 2 weeks.

The patient presented with an immature tooth with an open apex. So, the root canal system was obturated using a hydraulic condensation technique with a bioceramic sealer (Bioroot $\left.{ }^{\circ}\right)$. In fact, The Bioroot provides an excellent sealing ability, hydrophilic properties, and bioactivity. Thus, an impervious periapical barrier of the open apex and a good recovery of the lesion could be obtained. Also, as the root walls were so thin, hydraulic condensation allowed to avoid any possible risk of root fracture.

Apexification with a bioactive material barrier of Mineral Tioxide Aggregate (MTA) or Biodentine is also an option for open apices; however, it was not preferred in our case because the root walls were so thin. So, there was a high risk of fracture during vertical condensation of the apical plug.

Revascularization which is a novel method in the management of immature necrotic teeth with open apices can also be an option. Yet, considering the patient's general condition, his vital risk, the tooth history, and the large periapical lesion, it was not indicated.

Follow-up appointments and retro-alveolar radiographs are required to check the periapical lesion healing. In cases of persistence of the periapical lesion after a successful endodontic treatment and when non-surgical retreatment is inappropriate, endodontic surgery is indicated as the last option to remove the periapical pathology and to save the endodontically treated tooth.[14] In order to attain a successful outcome, the etiology of the persistent pathology should be determined.[7]

In fact, myriads of factors have been implicated in endodontic treatment failure. The usual factors are;
- Persistence of bacteria in periradicular tissues, in inaccessible apical areas or dental tubules, and in apically extruded debris require surgical intervention.[15] A study performed by Lin et al. involving 236 cases of endodontic failure found a correlation between the persistence of bacterial infection and periradicular lesions. [16(7)]

- The quality of root obturation influences the success rate of endodontic treatment. An inadequate or overextended root filling is an important factor for endodontic treatment failure. A study on a Spanish adult population in 2004 showed an association between increased incidence of periapical periodontitis and overextended root filling.[17] In fact, endodontic surgery is indicated when retreatment is impossible, when a bioceramic material is used as a sealer, or when there is a huge overfilling.

- An improper coronal seal can also be a factor for failure and may affect the prognosis of an endodontically treated tooth.

On the other hand, instrumentations fracture, root fracture, or root perforation may be a cause for orthograde treatment failure. So, endodontic surgery is necessary to explore and visualize the tooth root.[15]

In our case, faced with the persistence of periapical lesion and mucosal fistula despite the well filled root canal, an endodontic surgery was planned especially to explore the radiopacity detected into the lesion in the retro-alveolar radiograph to determine the possible cause of the treatment failure, and to remove the periapical pathology.

In fact, it was necessary to perform a 3D imaging. CBCT (cone beam computed topography) was required to have an idea about the nature of this radiopacity and to have additional information about the lesion size and the extent of bone loss.[14] Unfortunately, this was not possible due to the patient's limited financial means. Indeed, the main goal of apical surgery is to save teeth with periapical lesion, and which cannot be resolved by orthograde treatment. In fact, this goal is achieved by root-end section, rootend cavity preparation, and retrograde filling.[18] At first, the incision and the flap design should be chosen according to the clinical and radiographic parameters, such as the location and the extent of the lesion, the esthetic zone, the biotype, and the width of the gingival tissues.[18] In the anterior maxilla, submarginal incision is preferred to avoid gingival recession. However, if an apico-marginal communication is present or the lesion extends towards the alveolar crest, intrasulcular incision is a better choice.[18] After flap 
reflection, a small osteotomy should sometimes be performed to have access to the root. All the granulomatous and inflamed tissues should be completely debrided.[14]

In our case, during flap reflection, we found that the vestibular cortical bone overlying the tooth was resorbed and the root was exposed. During curettage, a dental structure inside the lesion corresponding to the opacity detected in the retro-alveolar radiograph was found. Odontoma with dental density and rounded edges could have been the cause of failure.

The next surgical step is root end resection. It is generally recommended to cut $3 \mathrm{~mm}$ from the root tip to remove the apical delta.[19] Resection should be as perpendicular as possible to the long axis of the root to reduce the number of exposed dentinal tubules and to ensure access to all the apical anatomy. The accessory canals which may be responsible for failure of endodontic treatment should be eliminated.[15]

After that, a successful hemostasis of the surgical site is very important for endodontic surgery success.[20] Bleeding control provides a better vision of the site. It facilitates root end resection and root end filling and reduces post-surgical hemorrhage and swelling. The hemostatic agent should be easy to handle. It should also be biocompatible and should not affect wound healing.[20] Various hemostatic agents, such as bone wax, collagen membranes, ferric sulfate, and epinephrine impregnated gauzes are proposed depending on their characteristics, advantages, and limits.[18] Plytetrafluroetylene (PTFE) strips are also proposed. They all offer an efficient bleeding control.[20] Yet, due to lack of materials, we used impregnated gauze of local anesthetic with vasoconstrictor to control bleeding. Once hemorrhage control is achieved, a careful inspection of the cut root face is recommended. So, the exposed root surface and the resected root face are strained with $2 \%$ methylene blue to identify possible leakage areas, such as root fractures, accessory canals, isthmuses, and gaps between the existing root canal filling and the root canal walls. [20]

Nowadays, the use of surgical microscope is recommended to ensure a better inspection of the surgical site at high magnification with excellent illumination, to obtain a better visualization of dentinal cracks, perforations and isthmuses, and to detect un-negotiated accessory canals.[14]

The next major step is root end preparation. It is performed with ultrasonic tips which should be used at low power to reduce the risk of root fracture and it should be carried out with sterile saline.[18] The use of microtips is a major breakthrough in apical surgery which ensures fewer traumas and faster bone healing. In fact, their small configuration allows for a small osteotomy and for the exposure of fewer dentin tubules.[14] Root end preparation is followed by root end filling to provide an apical seal to the canal system.[15]

Numerous materials are suggested for use as retrograde filling, including amalgam, zinc oxide-eugenol, composite resins, zinc phosphate cements, and glass ionomers. However, the most commonly used materials are IRM or EBA (erythroxybenzoic acid).[21] Studies have revealed favorable outcomes when using these materials, such as absence of postoperative symptoms, and favorable periapical healing and bone formation. [14] These materials should be biocompatible, non-toxic, non-irritant and capable to stimulate periapical tissues regeneration.[21] They should also have good handling proprieties, short time setting, and good radioopacity.[17]

Bioceramic root end filling materials, such as MTA or Biodentine have currently shown a success rate of $86 \%-95 \%$ as retrograde filling materials. Numerous studies have shown that MTA is a gold standard as an osteoinductive material and that it has a better sealing ability. However, recent studies have shown that Biodentine has more favorable outcomes than MTA. It is more effective both histologically and radiographically. It provides a short setting time, the least marginal leakage, and good handling properties.[14]

Finally, the mucosal flap is reapposed with sutures and the tissues are compressed for 3-5min with gauzes. [14] A membrane of PRF can be placed into the cavity to replace the bone defect and to stimulate bone healing, thus achieving a better healing of the soft tissues. [22] Healing of the periapical lesion should be evaluated by clinical and radiographic checkups. In our case, the radiograph after 12 months showed an almost complete healing of the periapical lesion.

\section{Conclusion}

The connection between oral health and metabolic control of diabetes is commonly known and a relationship cause-effect is established. DKA is a life-threatening episode of diabetic decompensation. Dental infections are reported as a possible cause of DKA.

So, it is important to know how to break the infectious circles of dental infection/Diabetes to get control of the disease process. A long-term control should be ensured by maintaining good oral hygiene and regular follow-up visits. 
Financial Disclosure: Nil.

Conflict of Interest: None declared.

\section{References}

1. Weinert LS, Scheffel RS, Severo MD, et al. Precipitating factors of diabetic ketoacidosis at a public hospital in a middle-income country. Diabetes Res Clin Pract 2012;96(1):29-34 doi:10.1016/j. diabres.2011.11.006

2. Chandu A, Macisaac RJ, Smith AC, Bach LA. Diabetic ketoacidosis secondary to dento-alveolar infection. Int J Oral Maxillofac Surg 2002;31(1):57-59 doi:10.1054/ijom.2001.0140

3. Calliari LE, Almeida FJ, Noronha RM. Infections in children with diabetes. J Pediatr (Rio J) 2020;96 Suppl 1:39-46 doi:10.1016/j. jped.2019.09.004

4. Karunakaran JV, Abraham CS, Karthik AK, Jayaprakash N. Successful nonsurgical management of periapical lesions of endodontic origin: A conservative orthograde approach. J Pharm Bioallied Sci 2017;9(Suppl 1):S246-S251 doi:10.4103/jpbs. JPBS_100_17

5. Segura-Egea JJ, Castellanos-Cosano L, Machuca G, et al. Diabetes mellitus, periapical inflammation and endodontic treatment outcome. Med Oral Patol Oral Cir Bucal 2012;17(2):e356-e361 doi:10.4317/medoral.17452

6. Juncar RI, Precup AI, Juncar M. Odontogenic inflammatory lesions in patients with type 2 diabetes mellitus: A prospective study of 128 cases. Niger J Clin Pract 2020;23(3):298-303 doi:10.4103/njcp.njcp_112_19

7. Tabassum S, Khan FR. Failure of endodontic treatment: The usual suspects. Eur J Dent 2016;10(1):144-147 doi:10.4103/13057456.175682

8. Magee MF, Bhatt BA. Management of decompensated diabetes. Diabetic ketoacidosis and hyperglycemic hyperosmolar syndrome. Crit Care Clin 2001;17(1):75-106 doi:10.1016/s07490704(05)70153-6

9. Shahgoli S, Shapiro R, Best JA. A dentoalveolar abscess in a pediatric patient with ketoacidosis caused by occult diabetes mellitus: a case report. Oral Surg Oral Med Oral Pathol Oral Radiol Endod 1999;88(2):164-166 doi:10.1016/s1079-2104(99)70111-3

10. Wolfsdorf JI, Glaser N, Agus M, et al. ISPAD Clinical Practice Consensus Guidelines 2018: Diabetic ketoacidosis and the hyperglycemic hyperosmolar state. Pediatr Diabetes 2018;19 Suppl 27:155-177 doi:10.1111/pedi.12701

11. Segura-Egea JJ, Martín-González J, Castellanos-Cosano L. Endodontic medicine: connections between apical periodontitis and systemic diseases. Int Endod J 2015;48(10):933-951 doi:10.1111/iej.12507
12. Segura-Egea JJ, Cabanillas-Balsera D, Jiménez-Sánchez MC, Martín-González J. Endodontics and diabetes: association versus causation. Int Endod J 2019;52(6):790-802 doi:10.1111/ iej. 13079

13. Ghorbanzadeh S, Ashraf H, Hosseinpour S, Ghorbanzadeh F. Nonsurgical management of a large periapical lesion: A case report. Iran Endod J 2017;12(2):253-256 doi:10.22037/ iej.2017.49

14. Showkat I, Sinha AA, Chaudhary S, Ghaus MA. Surgical intervention: Saviour of a failed root canal treatment. Int J Appl Dent Sci 2019;5(4):91-94

15. The Royal College of Surgeons, Guidelines for surgical endodontics. Version 2 (2012). Available at: https://www.rcseng.ac.uk/-/ media/files/rcs/fds/publications/surgical_endodontics_2012.pdf Accessed 05 May 2021

16. Lin LM, Skribner JE, Gaengler P. Factors associated with endodontic treatment failures. J Endod 1992;18(12):625-627 doi:10.1016/S0099-2399(06)81335-X

17. von Arx T. Apical surgery: A review of current techniques and outcome. Saudi Dent J 2011;23(1):9-15 doi:10.1016/j. sdentj.2010.10.004

18. Segura-Egea JJ, Jiménez-Pinzón A, Poyato-Ferrera M, VelascoOrtega E, Ríos-Santos JV. Periapical status and quality of root fillings and coronal restorations in an adult Spanish population. Int Endod J 2004;37(8):525-530 doi:10.1111/j.13652591.2004.00826.x

19. Abusrewil SM, McLean W, Scott JA. The use of Bioceramics as root-end filling materials in periradicular surgery: A literature review. Saudi Dent J 2018;30(4):273-282 doi:10.1016/j. sdentj.2018.07.004

20. Peñarrocha-Oltra $D$, Soto-Peñaloza $D$, Peñarrocha-Diago $M$, Cervera-Ballester J, Cabanes-Gumbau G, Peñarrocha-Diago M. Hemostatic agents in endodontic surgery of maxillary molars: A randomized controlled pilot study of polytetrafluoroethylene (PTFE) strips as an adjunct to epinephrine impregnated gauze versus aluminum chloride. Med Oral Patol Oral Cir Bucal 2020;25(5):e634-e643 doi:10.4317/medoral.23652

21. Alshaikh A, Alkahtani M, Alghamdi A, Alghamdi Y, Alghamdi F. Comparative evaluation of the different retrograde filling materials Bioceramic, Biodentine and Mmineral Trioxide Aggregate for endodontic surgery: A systematic review. EC Dental Science 2019;18(8):1749-1755

22. Singh S, Singh A, Singh S, Singh R. Application of PRF in surgical management of periapical lesions. Natl J Maxillofac Surg 2013;4(1):94-99 doi:10.4103/0975-5950.117825 